\title{
Example-Based Machine Translation using Associative Processors
}

\author{
Eiichiro Sumita,${ }^{\dagger}$ Kozo Oi,${ }^{\dagger}$ Osamu Furuse,${ }^{\dagger}$ Hitoshi Iida ${ }^{\dagger}$ \\ and Tetsuya Higuchi ${ }^{\dagger \dagger}$
}

This paper proposes an Example-Based Approach (EBA) using Associative Processors (APs) for machine translation, especially speech-to-speech translation, that requires (1) high accuracy and (2) a quick response. EBAs translate by mimicking the best-match translation examples (hereafter, "examples"), which are derived from corpora. These approaches are known to perform structural disambiguation, target word selection, and whole translation accurately. Therefore, EBAs fulfill the first requirement. The second requirement is also fulfilled by an EBA using APs as follows. The central mechanism of EBAs, Example-Retrieval (ER), retrieves the examples most similar to the input expression from an example database. ER becomes the dominant component as the size of the example database increases. We have parallelized ER by using APs consisting of an Associative Memory and a Transputer. Experimental results show that ER can be drastically accelerated by our method. Moreover, a study of communication among APs and an extrapolation from the sustained performance of 10 APs demonstrate the scalability of our method against the size of the example database. Consequently, the EBA using APs meets the critical requirements of machine translation.

KeyWords: machine translation, example-based approach, parallel processing, associative memory, speech-to-speech translation

\section{Introduction}

Machine translation requires (1) high accuracy and (2) a quick response. First, there is no doubt that translation accuracy is important in almost any situation, and it is desirable to minimize human intervention such as pre-, inter-, and post-interaction between user and machine, especially in real-time applications such as interpreting telephony. Second, a practical throughput is important for machine translation with a realistic domain not only for processing a large-scale text but also for real-time applications.

This paper proposes an Example-Based Approach (EBA) using Associative Processors (APs). EBAs translate by mimicking the best-match translation examples (hereafter, "examples"), which are derived from corpora. EBAs are known to perform structural disambiguation,

† ATR Interpreting Telecommunications Research Laboratories

i† Electrotechnical Laboratory 
target expression selection and whole translation accurately. Therefore, these approaches fulfill the first requirement. The second requirement is also fulfilled by an EBA using APs as follows. The central mechanism of EBAs, Example-Retrieval (ER), retrieves the examples most similar to the input expression from an example database. ER becomes the dominant component as the size of the example database increases. In this work, ER has been parallelized by using APs consisting of an Associative Memory and a Transputer. Experimental results show that ER can be drastically accelerated by our method. Moreover, a study of communication among APs and an extrapolation from the sustained performance of 10 APs demonstrate the scalability of our method against the size of the example database. Consequently, the EBA using APs meets the critical requirements of machine translation.

Section 2 explains EBAs and a sentence translation system using an EBA. Section 3 analyzes the computational cost of EBA systems. Section 4 describes the acceleration achieved by using APs. Section 5 explains the scalability of our method. Finally, Section 6 touches on related research.

\section{Example-Based Approach}

First, the idea behind EBAs and their notable features are introduced. Then, a sentence translation model using an EBA is discussed.

\subsection{Idea and Features}

Novel models for NLP have been studied in recent years. These methods have been called Example-Based Approaches (EBAs) because they rely on linguistic examples, such as translation pairs, derived from corpora. In the early 1980s, Nagao gave the first proposal of an EBA for machine translation, which translates by mimicking the best-match translation examples, based on the fact that a human translates according to past translation experience (Nagao 1984). Since the end of the 1980s, large corpora and powerful computational devices have allowed us to achieve Nagao's model and to expand the model to deal with not only translation but also other tasks such as parsing.

EBAs surpass conventional approaches for NLP in several aspects. The following is a summary of observations made in previous papers.

First, EBAs are accurate in a restricted domain, if sufficient examples are prepared. ${ }^{1}$

1 An observation that the translation quality improves as the number of examples increases, was reported in our previous paper (Sumita and lida 1992b).

As explained below, thesauri give an appropriate semantic granularity for problems of natural language processing. In the pp-attachment problem, compared with a statistically-based approach utilizing the frequency 
These approaches can deal with such well known difficult problems as target expression selection (e.g. function words (Sumita and Iida 1992a, 1992b), noun phrases (Sato 1993a) and verb phrases (Sato 1991)) and disambiguation of pp-attachment (Sumita, Furuse, and Iida 1993). EBAs achieve high accuracy not only for these subproblems in machine translation but also for sentence translation (Furuse, Sumita, and Iida 1994). We have trained our Japaneseto-English sentence translation system with the translations of 825 sentences in a conference registration domain. These sentences cover basic expressions used in Japanese aptitude tests conducted by the government. These sentences were reviewed by Japanese linguists as well. The translation success rate for these 825 sentences (trained data) is about $98 \%$. In addition, we have tested 1,056 sentences (unseen data) in the same domain; the translation success rate is about $71 \%$.

Second, EBAs are robust. The best-match mechanism using the semantic distance is robust against incompleteness of system knowledge. Moreover, some deviations from conventional grammars, which are specific to spoken language, are handled well (Furuse and Iida 1992). For example, in spoken Japanese, particles such as wa, o, and ni are frequently omitted. The omission is recovered well by EBAs.

Third, EBAs can output a reliability score (a number indicating how much we can rely on the result) unlike conventional NLP. EBAs provide a semantic distance as a reliability score. In our previous experiment (Sumita and Iida 1992b) on the relationship between semantic distance and success rate, we found the tendency that the smaller the semantic distance, the better the quality.

Fourth, the central mechanism of EBAs is language-independent. So far, we have implemented Japanese-to-English translation and vice versa. Not only example-retrieval but also the whole transfer process is shared by both systems (Sobashima, Furuse, Akamine, Kawai, and Iida 1994).

\subsection{Mechanism of Sentence Translation using EBA}

Here, we describe a sentence translation model featuring an EBA. A sentence is translated by combining partial examples in such a way that they cover the sentence jointly. Since the examples have a primary role and the whole process is controlled by transfer, we call our

of word co-occurrences, an EBA that utilizes word co-occurrences, i.e., examples and a thesaurus can achieve higher accuracy with a much smaller corpus. This implies that best-match based on the thesaurus compensates for the well known low-frequency data problem. Namely, words themselves are too finegrained to get sufficient amounts of data. In the case-frame selection problem, Nagao (Nagao 1992) compared an example-based method that utilizes a thesaurus with a conventional method that utilizes semantic markers consisting of several categories. The former is significantly better than the latter. Namely, semantic markers are too coarse-grained to resolve semantic ambiguity. 
model Transfer-Driven Machine Translation (TDMT) (Furuse and Iida 1992; Furuse et al. 1994; Furuse and Iida 1994).

A translation example, which is a piece of the transfer knowledge, describes the correspondence between a source language expression (SE) and target language expressions (TEs) as follows:

$$
\begin{array}{ccc}
S E= & T E_{1} & \left(E_{11}, E_{12}, \ldots\right), \\
& : & : \\
& T E_{n} & \left(E_{n 1}, E_{n 2}, \ldots\right)
\end{array}
$$

$\mathrm{SE}$ and $\mathrm{TE}$ are represented by patterns consisting of variables and surface expressions. $E_{i j}$ for $T E_{i}$ represents the $j$-th example word list that embodies variables of the SE. For example, the correspondence between typical Japanese noun phrases of the form, "X no Y" and English noun phrases is described as follows ${ }^{2}$ :

$$
\begin{aligned}
\mathrm{X} \text { no } \mathrm{Y}=>\quad \mathrm{Y}^{\prime} \text { of } \mathrm{X}^{\prime} & ((\text { ronbun }[\text { paper }], \text { daimoku }[\text { title }]), \cdots), \\
\mathrm{Y}^{\prime} \text { for } \mathrm{X}^{\prime} & ((\text { hoteru }[\text { hotel }], \text { yoyaku }[\text { reservation }]), \cdots), \\
\mathrm{Y}^{\prime} \text { in } \mathrm{X}^{\prime} & ((\text { Kyooto }[\text { Kyoto }], \text { kaigi }[\text { conference }]), \cdots),
\end{aligned}
$$

When TDMT translates the Japanese noun phrase "Oosaka[Osaka] no paatii[party]," it retrieves the best-match in the transfer knowledge, i.e. $\mathrm{X}$ no $\mathrm{Y}=>\mathrm{Y}^{\prime}$ ' in $\mathrm{X}$ ' (Kyooto[Kyoto], kaigi[conference]). According to this "best-match," TDMT generates "party in Osaka" by substituting English nouns for the Japanese nouns.

TDMT utilizes the semantic distance calculation previously proposed (Sumita and Iida 1992b) to retrieve the best-match examples. Let us suppose that an input, $I$, and each example, $E_{i j}$, consist of $t$ words as follows:

$$
\begin{aligned}
I & =\left(I_{1}, \cdots, I_{t}\right) \\
E_{i j} & =\left(E_{i j 1}, \cdots, E_{i j t}\right)
\end{aligned}
$$

Then, the semantic distance between $I$ and $E_{i j}$ is calculated as follows:

$$
d\left(I, E_{i j}\right)=\sum_{k=1}^{t} d\left(I_{k}, E_{i j k}\right) \times W_{k}
$$

The semantic distance between words, $d\left(I_{k}, E_{i j k}\right)$, is defined according to a thesaurus (Section 4.2). The weight $W_{k}$ is the degree to which the word influences the target expression

$2 \mathrm{X}$ and $\mathrm{Y}$ are variables for Japanese nouns and $\mathrm{X}$ ' and $\mathrm{Y}$ ' are the English translations of $\mathrm{X}$ and $\mathrm{Y}$, respectively; "no" is an adnominal particle that corresponds to English prepositions such as "of," "for," and "in." j[e] represents a Japanese word, $\mathrm{j}$ and the literal English translation word, $e$. 
selection. ${ }^{3}$

The flow of transferring an SE to the most plausible $T E$ is as follows:

[a] The semantic distance from the input is calculated for all examples.

[b] The example with the minimum-distance from the input is retrieved.

[c] The corresponding $T E$ of the minimum-distance example is selected as the most plausible one for the SE.

Processes [a] and [b] combine to form Example-Retrieval (ER), the focus of this paper.

The top-level TDMT algorithm is as follows: first, it produces possible source structures in which the SEs of the transfer knowledge are combined to cover the input; second, TDMT produces the target structures by transferring each SE of the source structures to the most appropriate TE using ER and [c] above.

For example, suppose the input Japanese sentence is as follows:

"kaigi no toorokuryou wa annaisho ni kisaisa re teimasu"

[conference] [of] [registrationfee] [sub] [announcement] [in] [be listed]

The source structure shown in Figure 1 is produced by combining SEs such as (X no $\mathrm{Y}$ ), ( $\mathrm{X}$ wa $\mathrm{Y}$ ), and ( $\mathrm{X}$ ni $\mathrm{Y}$ ). The target structure shown in Figure 2 is produced according to the transfer knowledge. Finally, the following translation is obtained:

"The conference registration fee is listed in the announcement."

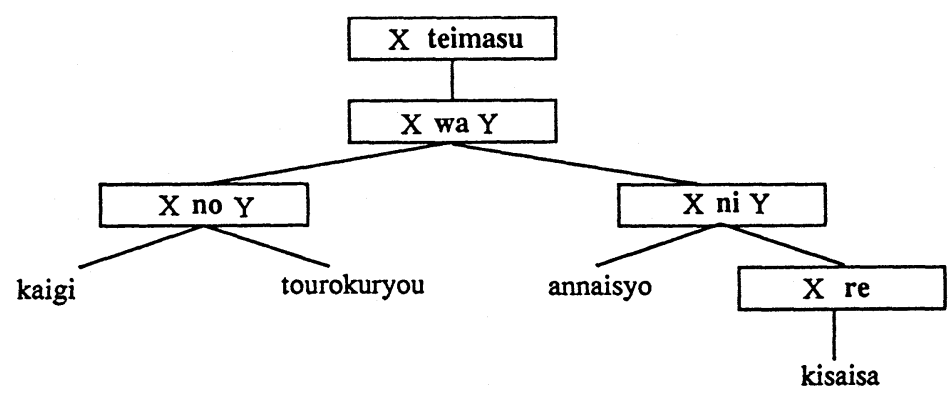

Fig. 1 Source structure

\section{Analysis of Computational Cost}

Example-Retrieval (ER), the key process of EBAs, retrieves the most similar examples by calculating the semantic distance between the input and examples. This section analyzes the 3 In the current prototype system of TDMT, $W_{k}$ is assigned to $1 / t$. 


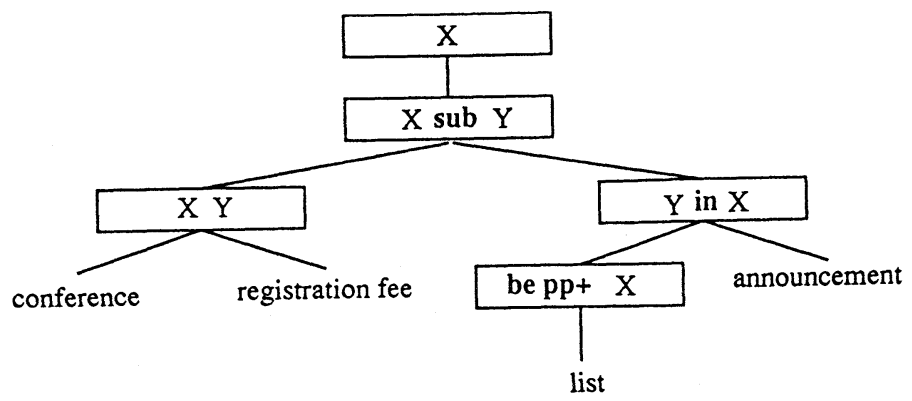

Fig. 2 Target structure

computational cost of ER by using TDMT, a typical EBA system introduced in the previous subsection. Although the analysis is done using TDMT, the findings are in general applicable to other EBA systems. First, we will show that ER is predominant in EBAs. Second, we investigate the ER cost in detail. Finally, we will present a rationale for using parallel machines to accelerate the ER of a large-scale EBA system.

\subsection{Example-Retrieval Cost of Prototype System}

Here, we show the timing figures of the prototype Japanese-to-English TDMT on a sequential machine (SPARCstation2) for 746 test sentences ${ }^{4}$ that are representative Japanese spoken sentences ${ }^{5}$.

Figure 3 shows that the increase in translation time is strongly related to the increase in ER time. The minimum, average and maximum translation time (ER time) per sentence is about 0.26 (0.001), 3.53 (2.49), and 23.28 (21.5) seconds, respectively.

\section{ER is the dominant component in EBAs because:}

(1) Even a single ER call is time-consuming. The ER time, $T$ (milliseconds), rises in proportion to the size of the example database, $N$, which is necessarily large. In the experiment, the following approximate relation was observed.

$$
T=0.65 N
$$

(2) ER is called many times in translating a single sentence. The number of ER calls, $C$, rises according to the sentence length, $L$. In our experiments, the following approximate relation between the number of ER calls and the sentence length was observed.

4 The average sentence length is about 10 words.

5 Of 825 sentences (described in Section 2.1), we used 746 sentences (about 90\%) in this experiment excluding sentences translated by exact-match of whole sentences, e.g., "arigatougozaimasu (Thank you very much)." Because exact-match sentences are translated momentarily, there is no need to accelerate their translation. 


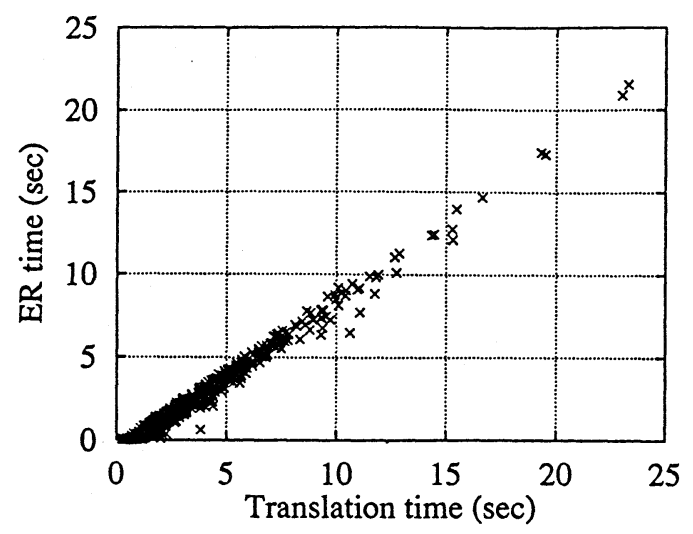

Fig. 3 ER time in sequential TDMT

$$
C=10^{\frac{L}{10}}
$$

The minimum, average and maximum number of ER calls per sentence is 1 , about 10 and 97 , respectively.

\subsection{Investigation of Example-Retrieval}

Table 1 shows that example-retrieval of frequent patterns, which include function words such as "wa," "no," "o," "ni" and "ga," takes up the majority of ER time in the Japaneseto-English version of TDMT. A rough explanation is as follows. The number of ER calls for a pattern is proportional to the frequency of the pattern. Since possible translations of a frequent pattern are, in general, diversified, the number of examples is roughly proportional to the frequency. Consequently, the computational cost of retrieving examples is proportional to the square of the frequency of the patterns.

In our experiment, the ER time for the top 10 patterns was found to account for nearly $96 \%$ of the entire ER time. There is little need to accelerate ER for other patterns. With this rationale, we decided to parallelize the ER for the top 10 patterns in the subsequent

Table 1 ER time rate per pattern

\begin{tabular}{|ccc|c|c|}
\hline \multicolumn{3}{|c|}{ Pattern } & Rate (\%) & Accumulative (\%) \\
\hline $\mathrm{X}$ & wa & $\mathrm{Y}$ & 25.20 & 25.20 \\
$\mathrm{X}$ & no & $\mathrm{Y}$ & 20.60 & 45.80 \\
$\mathrm{X}$ & o & $\mathrm{Y}$ & 19.61 & 65.41 \\
$\mathrm{X}$ & ni & $\mathrm{Y}$ & 11.13 & 76.54 \\
$\mathrm{X}$ & ga & $\mathrm{Y}$ & 8.90 & 85.44 \\
& $\vdots$ & & $\vdots$ & $\vdots$ \\
\hline
\end{tabular}


experiment.

In the E-to-J TDMT, the frequencies of patterns including function words, such as "at," "in," "on," "by," "to," and "of", are as large as those of J-to-E TDMT. Since they consume most of the ER time, we can take the same strategy to parallelize only the ER of frequent patterns.

\subsection{Example-Retrieval Cost of Large-Scale System}

We estimate the figures of a large-scale TDMT on two sequential machines: (1) the SPARCstation2 and (2) a future high-performance machine.

Let us estimate the size of the example database, $N$, for a large-scale system. In the prototype system, the vocabulary size is about 1,500 , and $N$ is 12,500 . Assuming that $N$ is in direct proportion to the square of the vocabulary size, then $N$ grows rapidly. Hereafter, we take into consideration the case $N=1,000,000$, i.e. about $9 * 9$ times larger than that of the prototype system.

For 14-word sentences ${ }^{6}$ we estimate figures of a large-scale TDMT based on those of the prototype TDMT. The ER time is proportional to $N$, while the time of the other processes are independent of $N$. Therefore, the expected translation time using a SPARCstation2 in the worst case is about $347.2(=\{\mathrm{ER}$ time $\}+\{$ time for the other processes $\}=\{4.32 \times 1,000,000 / 12,500\}+\{5.95-4.32\}=345.6+1.63)$ seconds. ${ }^{7} \mathrm{ER}$ consumes most of the CPU time, i.e. $99.5 \%$. This is clearly unacceptable for real-time applications.

Let us consider a much faster workstation, a 4,000 MIPS sequential machine ${ }^{8}$ instead of a SPARCstation2 (28.5 MIPS). The translation time is expected to be about 2.474 $(\simeq 347.2 \times 28.5 / 4,000)$ seconds. Of the 2.474 seconds, $(1)$ the ER is expected to take 2.462 seconds $(\simeq 345.6 \times 28.5 / 4,000)$, and $(2)$ the other processes are expected to consume 0.012 seconds $(\simeq 1.63 \times 28.5 / 4,000)$. Therefore, since it seems difficult to avoid disturbing natural communication with a sequential speech-to-speech translation system, we have decided to

6 To estimate more realistic figures than those using 746 test sentences, we use average sentences in a corpus. In our corpus (Ehara, Ogura, and Morimoto 1990), which contains about 13,000 sentences, the average sentence length is about 14 words. The average number of ER calls for all patterns is about 15, and the average number of ER calls for the top 10 patterns discussed in the previous subsection is about 6 . The translation time, ER time, and utterence time are about $5.95,4.32$, and 4.04 seconds on average, respectively.

7 This may seem larger than that expected according to the algorithm explained in Section 4.2 because the algorithm is rather simplified in some points. For example, it neglects the case where words are polysemous, i.e. have several different thesaurus codes and computing all combinations of codes requires much larger time.

8 A 4,000 MIPS sequential machine will be available in 10 years; this is based on the currently-available 200 MIPS machine and the current rate of increase for MIPS of about $35 \%$ per year (Hennessy and Patterson 1990). 
utilize parallelism to accelerate the ER.

\section{Acceleration Using Associative Processors}

Example-Retrieval (ER) is predominant in EBAs as described in Section 3.1; it does, however, have a nature suitable for parallel processing. We propose accelerating ER by using a parallel device, Associative Processor (AP). This section explains (1) AP; (2) ER on a single AP; and (3) ER on multiple APs.

\subsection{Associative Processors}

An Associative Processor (AP) is the processing element of the massively parallel machine, IXM2 (Higuchi, Furuya, Handa, Takahashi, Nishiyama, and Kokubu 1991). IXM2 has shown that a large Associative Memory (AM) works effectively as a SIMD device for AI applications where association and set operations dominate the total processing time. The AM not only features storage operations but also logical operations such as retrieving by content. Parallel search and parallel write are particularly important operations because they are sources of the AP's parallelism and are exploited in the ER algorithm, as will be explained in the next subsection. As specified in Table 2, each AP has a 4K-word AM and an INMOS T801 Transputer (TP). The AM is mapped into the memory space of the TP. A single AP allows one to perform $4 \mathrm{~K}$ search/write operations in parallel. APs are used in parallel TDMT as follows: (1) examples are loaded on the AM; (2) parallel communication and sequential process are done by the TP; (3) parallel search/write are triggered by the TP and executed by the AM.

Table 2 Components of an Associative Processor (AP)

- $4 \mathrm{~K} \times 40$ bit Associative Memory (AM), $375 \mathrm{~ns}$

- INMOS T801 Transputer (TP), $25 \mathrm{MHz}, 12.5 \mathrm{MIPS}$

$4 \mathrm{~KB}$ on-chip RAM, $40 \mathrm{~ns}$

$32 \mathrm{~K} \times 32$ bit SRAM, $80 \mathrm{~ns}$

4 serial links, $10 \mathrm{Mbits} / \mathrm{sec}$

\subsection{ER on a Single Associative Processor}

First, we explain the distance calculation part (process [a] in Section 2.2) of ExampleRetrieval for a single pattern on a single AP. Suppose $n$ is the number of examples and $t$ is the example length. The part can then be implemented as follows: $t$-time repetition of $n$-time summation of the distances between the $t$-th words of the input and the $n$-th example, 
multiplied by the $t$-th weights (see expression (1) in Section 2.2).

The distance between words is reduced to the distance between codes in a thesaurus. Each word is assigned a $k$-digit $l$-scale code that clearly represents the thesaurus hierarchy. In the thesaurus (Ohno and Hamanishi 1984) used in our experiment, a 3-digit decimal code is assigned. The semantic distance between codes is calculated according to Table 3. Sequential implementation of the distance calculation repeats word-by-word, accumulating the partial sums by computing, example-by-example, the conditions in Table 3 to yield $0,1 / 3,2 / 3$ or 1 .

Let us move on to the parallel implementation of the distance calculation part. The number of examples, $n$, is much larger than the example length of a pattern, $t$, so we have decided to parallelize the $n$-time summation. Table 4 outlines the elementary steps, i.e. parallel $n$ summation of $t$-th partial sums. [a1] to [a 4 ] in Table 4 correspond to [c1] to [c4] in Table 3. They are executed one-by-one using steps [i] to [iv].

Table 3 Semantic distance between thesaurus codes.

\begin{tabular}{|c|c|c|c|}
\hline \multicolumn{2}{|c|}{ Condition } & Example & Distance \\
\hline$[\mathrm{c} 1]$ & $C I_{1} C I_{2} C I_{3}=C E_{1} C E_{2} C E_{3}$ & 347,347 & 0 \\
\hline$[\mathrm{c} 2]$ & $C I_{1} C I_{2}=C E_{1} C E_{2}, C I_{3} \neq C E_{3}$ & $\mathbf{3 4 7 ,} \mathbf{3 4 6}$ & $1 / 3$ \\
\hline [c3 & $C I_{1}=C E_{1}, C I_{2} \neq C E_{2}$ & $\mathbf{3 4 7 ,} 337$ & $2 / 3$ \\
\hline$[\mathrm{c} 4]$ & $C I_{1} \neq C E_{1}$ & 347,247 & 1 \\
\hline
\end{tabular}

Table 4 Calculating semantic distance per word between input and all examples on a single Associative Processor (AP)

$X$ is the retrieval data and $Y$ is the distance.

[a1] Retrieve the cells whose distance from the input is 0.

Set $X=$ "all three digits of the input code" and $Y=0$, execute [i]-[iv].

[a2] Retrieve the cells whose distance from the input is $1 / 3$.

Set $X=$ "flag bit (0) and upper two digits of the input code" and $Y=1 / 3$, execute [i]-[iv].

[a3] Retrieve the cells whose distance from the input is $2 / 3$.

Set $X=$ "flag bit (0) and upper one digit of the input code" and $Y=2 / 3$, execute [i]-[iv].

[a4] Retrieve the cells whose distance from the input is 1.

Set $X=$ "flag bit (0)" and $Y=1$, execute [i]-[iii].

[i] Mark all cells which matches $X$ simultaneously on the AM.

[ii] Write 1 at the flag bit of all marked cells simultaneously on the AM to mask them out in the following retrieval.

[iii] Get the address of a marked cell and add $Y \times$ weight to a semantic distance variable on the TP that corresponds to the address. Repeat this process until the marked cells run out.

[iv] Clear all marks on the AM. 


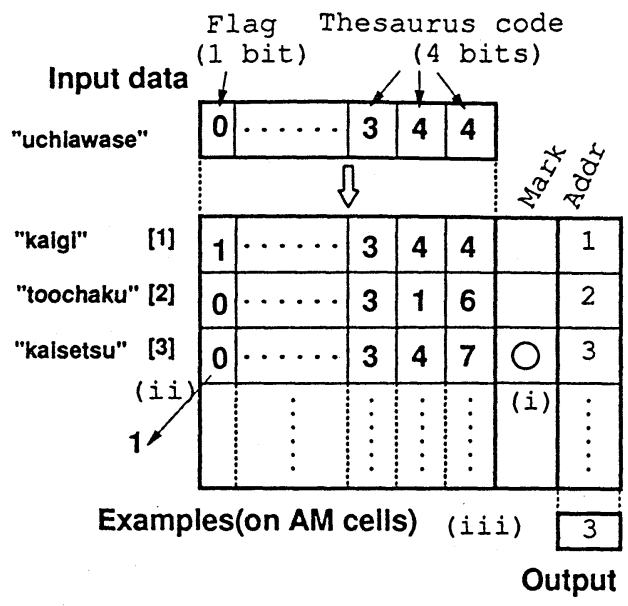

Fig. 4 Illustration of semantic distance calculation using Associative Memory (AM)

Figure 4 illustrates a portion of the AM; the input data is 344, which is the thesaurus code of the word "uchiawase[meeting]." Each code of the examples is stored in the AM. 344, 316, 347 represent the codes for "kaigi[conference]," "toochaku[arrival]," "kaisetsu[explanation]" of the examples. Figure 4 shows steps [i] to [iii] of [a2] after [a1] is completed. Step [i] marks the third cell, step [ii] writes 1 at the flag bit of the third cell, and step [iii] gets 3, i.e. the address of the marked cell. Steps [i], [ii] and [iv] are done for all cells by the parallel search/write operations of the AM in a constant time. Step [iii] is done in the TP and repeated by the number of marked cells. Parallel implementation identifies examples not by repeating but by executing the match and conditional branch simultaneously to perform step [iii] with given values, i.e. $0,1 / 3,2 / 3$ or 1 .

TP sequentially performs the process that finds the minimum of the distances betreen the input and examples and locates the examples whose distance is minimum (process [b] in Section 2.2) in Example-Retrieval.

We have already successfully conducted a preliminary experiment of ER for a single pattern on a single AP (Sumita, Oi, Furuse, Iida, Higuchi, Takahashi, and Kitano 1993). In the experiment, the AP outperformed state-of-the-art machines by using the parallel search/write functions of the AM. ${ }^{9}$

9 An investigation on a single ER call revealed that the AM consumes at most a minute order of time, i.e. 10 microseconds, for $4 \mathrm{~K}$ parallel operations and that the TP consumes time on the order of 10 milliseconds. In other words, although ER is drastically accelerated by exploiting the AM's parallelism, the remaining sequential processes of ER, i.e. step [iii] of process [a] and process [b], are predominant for ER on a single AP. We can achieve greater acceleration because improving the performance of the TP directly decreases the ER time. 


\subsection{ER on Multiple Associative Processors}

According to the performance analysis in subsection 3.2, we targeted only the top 10 patterns in the experiment for multiple patterns on multiple APs. The number of examples for each pattern of prototype system was at most about 1,000, and in our implementation each AP could load about 1,000 examples; thus, 10 APs were sufficient for the experiment. The 10 APs $\left(\mathrm{AP}_{1}, \mathrm{AP}_{2}, \cdots, \mathrm{AP}_{10}\right)$ and the root $\mathrm{TP}$, which was built into the HOST (the SPARCstation2), were connected in a tree configuration as shown in Figure 5. ${ }^{10}$

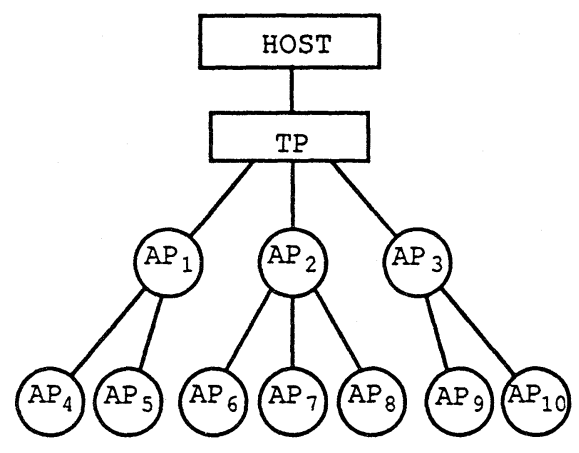

Fig. 5 Configuration of TDMT using 10 Associative Processors (APs)

The ER algorithm using multiple APs(Higuchi, Handa, Takahashi, Furuya, Iida, Sumita, Oi, and Kitano 1994; Oi, Sumita, Furuse, Iida, and Higuchi 1994) is shown in Table 5. This algorithm implements ER by distributing examples onto multiple APs, retrieving examples on each AP in parallel and merging all results.

We compared two different methods of loading examples as follows (see Figure 6):

Homo-loading (HM) Each AP is loaded with examples of a pattern.

Hetero-loading (HT) Each AP is loaded with examples that are equally distributed to all APs, of 10 different patterns.

Figures 7 and 8 plot the acceleration of ER for TDMT using multiple APs over sequential TDMT with the two methods. The acceleration with the HT method is greater than that with the HM method because the sequential part, i.e. step [iii] of process [a] and process [b], is proportional to the number of examples of the pattern in question on the APs and because

10 The tree configuration is trinary because each TP has four serial links, as shown in Table 2. The root TP and the HOST are connected through an SBus. The TDMT main program is written in Lisp and is executed on the HOST (SPARCstation2). The ER routine is programmed in Occam2 and executed on the APs and the root TP. 
Table 5 ER Algorithm using multiple Associative Processors (APs)

[m1] Send input data from the HOST to the root TP.

[m2] Send the input data sent from the upper directly-connected processors to the lower directlyconnected APs in parallel.

[m3] Execute ER locally on each AP in parallel (the minimum distance and the examples whose distance is minimum in an AP are obtained).

[m4] Send the ER result, after merging its own result and the results sent from lower directlyconnected APs in parallel, to the upper directly-connected processors.

[m5] Send the merged result from the root TP to the HOST.

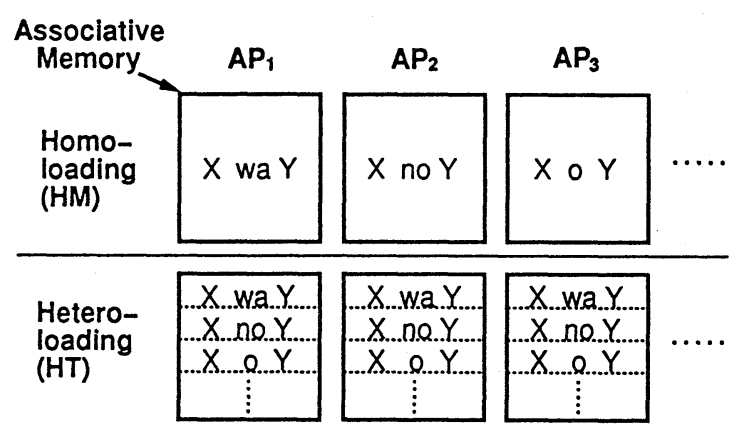

Fig. 6 Two methods of loading examples

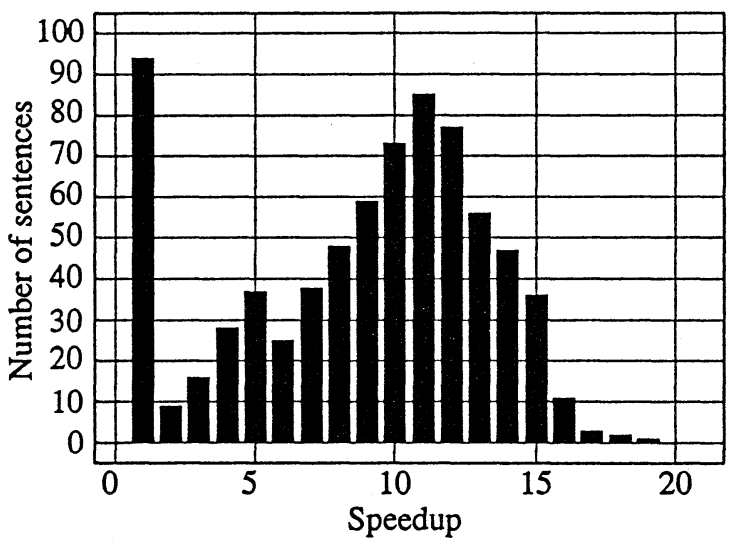

Fig. 7 Acceleration of ER (HM) over sequential TDMT

the numbers in HT are much less than those in HM. The sentence-by-sentence acceleration changes largely depending on to what extent the sentence calls up ER for the top 10 patterns. Needless to say, about 90 sentences are not accelerated because they do not include the ER 


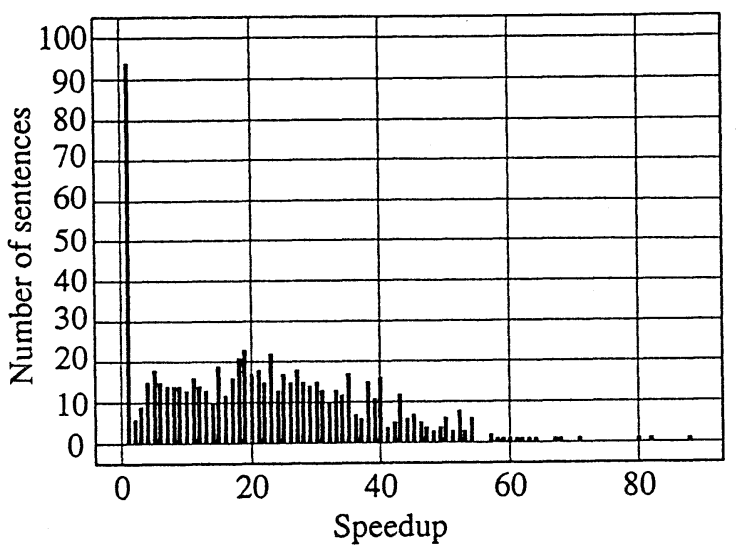

Fig. 8 Acceleration of ER (HT) over sequential TDMT

for the top 10 patterns. With the HT method, the average acceleration of ER is about 16.4 $(\simeq 2.4897 / 0.1522=\{$ the average time (seconds) per sentence in the sequential TDMT $\} /\{$ the average time (seconds) per sentence in the HT method\}).

Table 6 summarizes the performance of prototype TDMTs (on the SPARCstation2 in Section 3.1 and on APs with the SPARCstation2 in this section). These results do not look so striking. Because the prototypes are rather small-scale, there is no urgent need to achieve acceleration even by parallelizing TDMT. However, sequential implementation cannot scale up, as discussed in Section 3.3. On other hand, parallel TDMT on multiple APs exhibits clear scalabilty as will be shown in the next section. Table 7 gives a time analysis of a single ER call on a prototype parallel TDMT using the HT method (referring to the algorithm of Table 5). (a) and (b) are controlled not by the size of the example database but by the depth of the AP tree because an AP can send data to those APs directly-connected in parallel (e.g. $\mathrm{AP}_{2} \rightarrow \mathrm{AP}_{6}, \mathrm{AP}_{2} \rightarrow \mathrm{AP}_{7}, \mathrm{AP}_{2} \rightarrow \mathrm{AP}_{8}$ ) and vice versa ${ }^{11}$. Thus, (a) and (b) are the unit times between two APs $\times$ the tree depth, 2 (see Figure 5). The unit times between two APs for (a) and (b) are fairly small, i.e. several tens of microseconds, because the data size is only several tens of bytes and the bandwidth of the link between two TPs is 10 Mbits/sec. Thus the sum, (c), communication time among APs, is controlled by the tree depth and a small coefficient. Step [m3] can be regarded as a constant operation because even if the total number of examples increases, the number of examples for each AP is roughly invariant. Step [m1] is of a constant-time because it only does operations related to

11 This is because a TP can send data to those TPs directly-connected in parallel and the APs consist of an AM and a TP 
Table 6 Time comparison of prototype TDMTs

\begin{tabular}{|c|c|}
\hline \multicolumn{2}{|c|}{ number of examples: 12,500} \\
\hline sequential & parallel \\
\hline $\begin{array}{c}\text { SPARCstation2 } \\
(28.5 \text { MIPS })\end{array}$ & 10AP+SPARCstation2 \\
\hline $3.53(2.49+1.04)$ & $1.19(0.15+1.04)$ \\
\hline NB: A (B+C): A = Translation, $\mathrm{B}=\mathrm{ER}$ and $\mathrm{C}=$ Other
\end{tabular}
NB: $A(B+C): A=$ Translation, $B=E R$ and $C=O$ ther

Table 7 Time of steps in a single ER call

\begin{tabular}{|l|c|}
\hline \multicolumn{1}{|c|}{ Steps in ER } & msec \\
\hline (a) distribute the input data through all APs (step [m2]) & $0.10\left(0.05^{*} 2\right)$ \\
(b) merge \& collect the result from all APs (step [m4]) & $0.12\left(0.06^{*} 2\right)$ \\
(c) communicate among APs (=(a)+(b)) & $0.22\left(0.11^{*} 2\right)$ \\
(d) (1) ER (step [m3]) and (2) communicate between the HOST and the root & 13.15 \\
TP (steps [m1] and [m5]) & \\
\hline
\end{tabular}

NB: Each time is the average figure per ER call when translating 746 sentences.

input sending. Step [m5] can be regarded as a constant operation because it depends on the size of the ER result, which does not sigificantly change. Consequently, time (d) is invariant.

\section{Scalability toward Large-Scale System using Associa- tive Processors}

The experiment in the previous section and the estimation in this section show that ER on multiple APs is clearly scalable against the size of the example database. That is, even though the size of the example database increases by nearly two figures from the prototype system, the ER time increases slightly and is applicable to real-time applications.

\subsection{Configuration}

Let us estimate the tree depth of the AP tree necessary to load examples of a large-scale system (Section 3.3). The tree depth is the minimum D satisfying the inequality:

$$
\sum_{x=1}^{\mathrm{D}} 3^{x} \geq \frac{N}{12,500} \times 10
$$

The right hand side is the number of APs. Because prototype TDMT $(N=12,500)$ requires 10 APs, a large-scale TDMT $(N=1,000,000)$ requires 800 APs. Thus, the tree depth, D, is 6. Figure 9 shows 800 APs in a trinary tree structure. We assume a 4,000 MIPS sequential 
workstation as the host here because it is not conclusively determined whether those other than ER is suitable for parallel computation. This is a hybrid acceleration in that (1) the predominant part, ER, is accelerated by APs; (2) the other part is accelerated by a high performance sequential machine (4,000 MIPS).

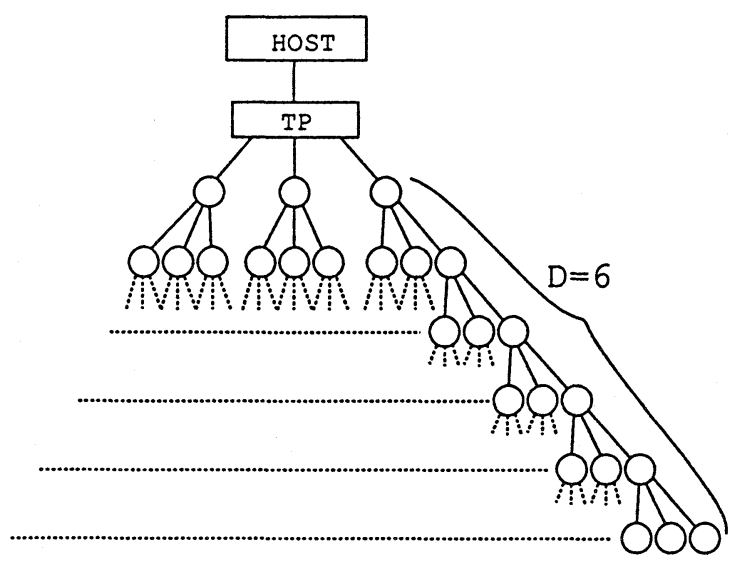

Fig. 9 Configuration of large-scale TDMT using 800 APs

\subsection{Estimation}

We estimate the time for translating the 14-word sentences based on the extrapolated figures for the 4,000 MIPS machine shown in Section 3.3. The translation time is divided into parts (a) to (i), as shown in Table 8. (a) to (e) are explained according to Table 7. Recall that the tree depth of 800 APs is 6 (Figure 9) and the number of ER calls per 14-word sentence is 6.

The distribution time to all APs $(\mathrm{a})$ is nearly $1.8(=0.05 \times 6 \times 6)$ milliseconds because the average distribution time between directly-connected APs is about 0.05 milliseconds, the tree depth is 6 , and the average number of ER calls is about 6 . In the same way, the collection time from all APs $(\mathrm{b})$ is about $2.2(=0.06 \times 6 \times 6)$ milliseconds. Thus, the total communication time among APs (c) is about $1.8+2.2=4.0$ milliseconds. The time (d) involving (1) ER on each AP and (2) communication between the HOST and the root TP is not related to the tree depth, so it is simply multiplied by the number of ER calls, 6 , yielding $78.9=13.15^{*} 6$ milliseconds. Accordingly, the ER time for only the top 10 patterns (e) is 82.9 milliseconds.

(f) to (i) are estimated based on (e) and the 4,000 MIPS's estimation in Section 3.3. The 
Table 8 Estimation of translation time on large-scale parallel TDMT

\begin{tabular}{|c|c|c|c|c|}
\hline \multicolumn{5}{|c|}{ (i) translation } \\
\hline \multicolumn{5}{|c|}{193} \\
\hline \multicolumn{4}{|c|}{ (g) ER } & (h) other on HOST \\
\hline \multicolumn{4}{|c|}{181} & \multirow[t]{7}{*}{12} \\
\hline \multicolumn{3}{|c|}{ (e) ER for top 10} & $\begin{array}{l}\text { (f) ER for other than } \\
\text { top } 10 \text { on HOST }\end{array}$ & \\
\hline \multicolumn{3}{|c|}{82.9} & \multirow[t]{5}{*}{98} & \\
\hline (c) comm. an & nong APs & $\begin{array}{l}\text { (d) (1) ER and }(2) \text { comm } \\
\text { between the HOST and } \\
\text { the root TP }\end{array}$ & & \\
\hline 4.0 & & \multirow[t]{3}{*}{78.9} & & \\
\hline (a) distribute & (b) collect & & & \\
\hline 1.8 & 2.2 & & & \\
\hline
\end{tabular}

NB: Milliseconds per 14-word sentence

ER time for patterns other than the top 10 patterns (f) is 98 milliseconds ${ }^{12}$ because the entire ER time is estimated to be 2462 milliseconds (in Section 3.3), and ER for other than the top 10 patterns consumes $4 \%$ of the time (in Section 3.2 ). (g) is the sum of (e) and (f). (h) is 12 milliseconds (Section 3.3). The grand sum, i.e. the translation time of 14 -word sentences (i), is 193 milliseconds.

Table 9 summarizes the performance of large-scale systems.

(1) The ER time of the SPARCstation2 in the worst case is unacceptably large (345.6 seconds). The APs accelerated ER from 345.6 seconds to 0.18 seconds.

(2) The time for processes other than ER is effectively reduced from $1.63 \mathrm{sec}$ to 0.01 inversely proportional to the increase in MIPS. This part is not necessarily suitable for APs and is tractable with a high-performance sequential machine.

Table 9 Time comparison of large-scale TDMTs

\begin{tabular}{|c|c|}
\hline \multicolumn{2}{|c|}{ number of examples: $1,000,000$} \\
\hline sequential & parallel \\
\hline $\begin{array}{c}\text { SPARCstation2 } \\
(28.5 \text { MIPS })\end{array}$ & 800AP+sequential machine \\
\hline 347.000 MIPS $)$
\end{tabular}

NB: $A(B+C): A=$ Translation, $B=E R$ and $C=$ Other Seconds per 14-word sentence

\footnotetext{
12 So far, we have assumed that only ER for the top 10 patterns is parallelized. We can achieve more acceleration if we include other frequent patterns to minimize (f) under the condition that the parallelizing overhead is not exceeded.
} 


\section{Related Research}

\subsection{Massively Parallel Natural Language Processing}

Up to now, some systems using a massively parallel machine in the field of natural language processing, such as a parsing system (Kitano and Higuchi 1991b) and translation systems, e.g. ASTRAL (Kitano and Higuchi 1991a), MBT3n (Sato 1993b), have been proposed. They have demonstrated good performance; nonetheless, they differ from our proposal. The first two systems, although they use APs, use a different mechanism for their natural language tasks, i.e. they do not calculate the semantic distance but propagate markers through a semantic network. The last system deals with a translation subproblem, i.e. translating not sentences but noun phrases (technical terms), using a different mechanism based on matching and similarity on a MIMD machine.

\subsection{Speech-to-Speech Translation}

Challenging research on speech-to-speech translation began in the mid-1980s. Such research has brought about several prototype systems (Morimoto, Takezawa, Yato, Sagayama, Tashiro, Nagata, and Kurematsu 1993; Kitano 1991; Waibel, Jain, McNair, Saito, Hauptmann, and Tebelskis 1991; Rayner, Alshawi, Bretan, Carter, Digalakis, Gamback, Kaya, Karlgren, Lyberg, Pulman, Price, and Samuelsson 1993; Hatazaki, Yoshida, Okumura, Mitome, Watanabe, Fujimoto, and Narita 1992). However, no large-scale system capable of responding in real-time has emerged. Speech-to-speech translation consists of three processes, i.e. speech recognition, spoken language translation and speech synthesis. There are two possible models for speech-to-speech translation: (1) a simultaneous model where subsequent processes start after a slight delay and overlap each other; and (2) a sequential model where processes start after their preceding process is completed. Unfortunately, state-of-the-art NLP technologies do not allow us to adopt the simultaneous model; thus, each component in the sequential model should be accelerated as much as possible.

\section{Concluding Remarks}

An EBA using APs has been proposed. The translation quality of EBAs is high according to our previous experiment. Using APs, EBAs have been drastically accelerated with a good scalability against the size of the example database. Consequently, an EBA using APs meets the critical requirements necessary to break through the limitations of conventional machine 
translation, especially for spoken language translation.

The acceleration and scalability of our method using APs are due to the following: (1) ER strongly dominates EBAs; (2) The AM's parallelism is employed for ER on a single AP; (3) the communication time among APs is controlled by the tree depth, which is fairly shallow.

EBAs using APs have desirable features suitable for integration with speech recognition: (a) high accuracy; (b) robustness; (c) output of a reliability score; and (d) a quick response. Tightly coupling our model and speech recognition might make possible real-time speech-tospeech translation in the future.

\section{Acknowledgement}

The authors wish to acknowledge the help received from Dr. Yasuhiro YAMAZAKI (the President of ATR-ITL), Dr. Sakutaro TOMIYAMA (the Director of ETL), Dr. Hiroaki KITANO (CMU), and Kadokawa-shoten (the publisher of Ruigo-Shin-Jiten).

\section{Reference}

Ehara, T., Ogura, K., and Morimoto, T. (1990). "ATR Dialogue Database." In Proc. of ICSLP'90, Vol. 2, pp. 1093-1096.

Furuse, O., and Iida, H. (1992). "Cooperation Between Transfer and Analysis in ExampleBased Framework." In Proc. of COLING'92, pp. 645-651.

Furuse, O., and Iida, H. (1994). "Constituent Boundary Parsing for Example-Based Machine Translation." In Proc. of COLING'94, pp. 105-111.

Furuse, O., Sumita, E., and Iida, H. (1994). "Transfer Driven Machine Translation Utilizing Empirical Knowledge." Transactions of Information Processing Society of Japan, 35(3), 414-425.

Hatazaki, K., Yoshida, K., Okumura, A., Mitome, Y., Watanabe, T., Fujimoto, M., and Narita, K. (1992). "A Japanese-English bidirectional automatic interpretation system: INTERTALKER." In 44th convention of IPSJ , 6P-5.

Hennessy, J. L., and Patterson, D. A. (1990). Computer Architecture: A Quantitative Approach. Morgan Kaufmann.

Higuchi, T., Furuya, T., Handa, K., Takahashi, N., Nishiyama, H., and Kokubu, A. (1991). "IXM2 : A Parallel Associative Processor." In Proc. of the 18th International Symposium on Computer Architecture.

Higuchi, T., Handa, K., Takahashi, N., Furuya, T., Iida, H., Sumita, E., Oi, K., and Kitano, H. (1994). "The IXM2 Parallel Associative Processor for AI." IEEE COMPUTER, 27(11), 53-63. 


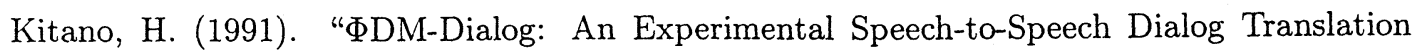
System." IEEE Computer, 24(6), 36-50.

Kitano, H., and Higuchi, T. (1991a). "High Performance Memory-Based Translation on IXM2 Massively Parallel Associative Memory Processor." In Proc. of AAAI'91, Vol. 1, pp. 149-154.

Kitano, H., and Higuchi, T. (1991b). "Massively Parallel Memory-Based Parsing." In Proc. of IJCAI'91, pp. 918-924.

Morimoto, T., Takezawa, T., Yato, F., Sagayama, S., Tashiro, T., Nagata, M., and Kurematsu, A. (1993). "ATR's Speech Translation System: ASURA." In Proc. of EUROSPEECH'93, pp. 1291-1294.

Nagao, M. (1984). "A Framework of a Mechanical Translation between Japanese and English by Analogy Principle." In Elithorn, A., and Banerji, R. (Eds.), Artificial and Human Intelligence, pp. 173-180. North-Holland.

Nagao, M. (1992). "Some Rationales and Methodologies for Example-Based Approach." In Proc. of FGNLP'92, pp. 82-94.

Ohno, S., and Hamanishi, M. (1984). Ruigo-Shin-Jiten. Kadokawa.

Oi, K., Sumita, E., Furuse, O., Iida, H., and Higuchi, T. (1994). "Real-Time Spoken Language Translation Using Associative Processors." In Proc. of ANLP'94, pp. 101-106.

Rayner, M., Alshawi, H., Bretan, I., Carter, D., Digalakis, V., Gamback, B., Kaya, J., Karlgren, J., Lyberg, B., Pulman, S., Price, P., and Samuelsson, C. (1993). "A Speech to Speech Translation System Built from Standard Components." In Proc. of the Workshop on Human Language Technology, pp. 217-222. ARPA.

Sato, S. (1991). Example-Based Machine Translation. Ph.D. thesis, Kyoto University.

Sato, S. (1993a). "Example-Based Translation of Technical Terms." In Proc. of the TMI'93, pp. 58-68.

Sato, S. (1993b). "MIMD Implementation of MBT3." In Proc. of the Workshop on Parallel Processing for Artificial Intelligence, pp. 28-35. IJCAI'93.

Sobashima, Y., Furuse, O., Akamine, S., Kawai, J., and Iida, H. (1994). "A Bidirectional, Transfer-Driven Machine Translation System for Spoken Dialogues." In Proc. of COLING'94, pp. 64-68.

Sumita, E., Furuse, O., and Iida, H. (1993). "An Example-Based Disambiguation of Prepositional Phrase Attachment." In Proc. of TMI'93, pp. 80-91.

Sumita, E., and Iida, H. (1992a). "Example-Based NLP Techniques - A Case Study of Machine Translation." In Proc. of Statistically-Based NLP Techniques Workshop (AAAI'92). 
Sumita, E., and Iida, H. (1992b). "Example-Based Transfer of Japanese Adnominal Particles into English." IEICE TRANS. INF. \& SYST., E75-D(4), 585-594.

Sumita, E., Oi, K., Furuse, O., Iida, H., Higuchi, T., Takahashi, N., and Kitano, H. (1993). "Example-Based Machine Translation on Massively Parallel Processors." In Proc. of IJCAI'93, Vol. 2, pp. 1283-1288.

Waibel, A., Jain, A., McNair, A., Saito, H., Hauptmann, A., and Tebelskis, J. (1991). "JANUS: A Speech-to-speech Translation Using Connectionist and Symbolic Processing Strategies." In Proc. of ICASSP'91, Vol. 2, pp. 793-796. 
Eiichiro Sumita: Eiichiro Sumita received the B.E. and M.E. degrees in computer science from University of Electro-Communications, in Tokyo, in 1980 and 1982 respectively. He is a senior researcher of ATR Interpreting Telecommunications Research Laboratories. His research interests include natural language processing, information retrieval and parallel processing. $\mathrm{He}$ is a member of IPSJ and IEICE.

Kozo Oi: Kozo Oi received the B.E. degree in electronic engineering from Doshisha University, Kyoto, Japan, in 1984. He joined SANYO Electric Co., Ltd. in 1984. He has been on loan to ATR since 1992. He is now a researcher of ATR Interpreting Telecommunications Research Laboratories. His research interests are natural language processing and parallel processing. He is a member of IPSJ.

Osamu Furuse: Osamu Furuse received the B.E. and M.E. degrees in information engineering from Kyushu University, Fukuoka, Japan, in 1982 and 1984 respectively. From 1984 to 1990, he worked at NTT Electrical Communication Laboratories. He has been on loan to ATR since 1990. $\mathrm{He}$ is now a senior researcher of ATR Interpreting Telecommunications Research Laboratories. He has been engaged in natural language processing research.

Hitoshi Iida: Hitoshi Iida received a BS and an MS in mathematics in 1972 and 1974 from Waseda University. He joined the Electorical Communication Laboratories of Nippon Telegaraph and Telephone in 1974 and has temporarily moved to ATR Interpreting Telecommunications Research Labs. His interests are dialogue comprehension and speech translation.

Tetsuya Higuchi: Tetsuya Higuchi received his $\mathrm{BS}, \mathrm{ME}$, and $\mathrm{PhD}$ degress in 1978,1980 , and 1984 from Keio University. He heads the computational models section at Electrotechnical Laboratory. His research interests include parallel processing and associative processing. He is a member of IPSJ.

(Received September 20, 1994 )

(Revised February 13, 1995)

(Accepted March 3, 1995 ) 